\title{
EXTENDING THE EHRESMANN-SCHEIN-NAMBOORIPAD THEOREM
}

\author{
CHRISTOPHER HOLLINGS
}

\begin{abstract}
We extend the ' $V$-premorphisms' part of the Ehresmann-ScheinNambooripad Theorem to the case of two-sided restriction semigroups and inductive categories, following on from a result of Lawson (1991) for the 'morphisms' part. However, it is so-called ' $\wedge$-premorphisms' which have proved useful in recent years in the study of partial actions. We therefore obtain an Ehresmann-Schein-Nambooripad-type theorem for (ordered) $\wedge$-premorphisms in the case of two-sided restriction semigroups and inductive categories. As a corollary, we obtain such a theorem in the inverse case.
\end{abstract}

\section{INTRODUCTION}

In their study of $E$-unitary covers for inverse semigroups, McAlister and Reilly [17] made the following definitions:

Definition 1.1. [17, Definition 3.4] Let $S$ and $T$ be inverse semigroups. A $(\vee, i)$ premorphism is a function $\theta: S \rightarrow T$ such that

$(\mathrm{V} 1)(s t) \theta \leq(s \theta)(t \theta)$.

Definition 1.2. [17, Definition 4.1] Let $S$ and $T$ be inverse semigroups. A $(\wedge, i)$ premorphism is a function $\theta: S \rightarrow T$ such that 1

$(\wedge 1)(s \theta)(t \theta) \leq(s t) \theta$;

$(\wedge 2)^{\prime}(s \theta)^{-1}=s^{-1} \theta$.

(We note that in [17], a $(\vee, i)$-premorphism was termed a $v$-prehomomorphism, whilst in [16, p. 80], it is termed simply a prehomomorphism. In [17, a $(\wedge, i)$ premorphism was called a $\wedge$-prehomomorphism; in [16, p. 80], it is called a dual prehomomorphism.)

Date: August 2, 2018.

2000 Mathematics Subject Classification. 20 M 18, 20 L 05, 20 M 15.

Key words and phrases. restriction semigroup, inverse semigroup, inductive category, inductive groupoid, premorphism.

This work was completed as part of Project POCTI/0143/2007 of CAUL, financed by FCT and FEDER, and also as part of FCT post-doctoral research grant SFRH/BPD/34698/2007. Thanks must go to both Mark Lawson and Victoria Gould for a number of useful comments.

${ }^{1}$ We place $\mathrm{a}^{\prime}$ on condition $(\wedge 2)^{\prime}$, as we will shortly replace this condition by a weaker one and we wish to reserve the label $(\wedge 2)$ for that. 
These functions were central to McAlister and Reilly's constructions: see their Theorems 3.9 and 4.5 [17].

In the present paper, we will study generalisations of the functions of Definitions 1.1 and 1.2 in the case of so-called two-sided restriction semigroups (until recently, termed weakly E-ample semigroups). These are an extremely natural class of semigroups which generalise inverse semigroups and which arise from partial transformation monoids in a manner analogous to the way in which inverse semigroups arise from symmetric inverse monoids. The concrete description of such a semigroup is as follows. Let $\mathcal{P} \mathcal{T}_{X}$ denote the collection of all partial mappings of a set $X$, i.e., all mappings $A \rightarrow B$, where $A, B \subseteq X$. We compose elements of $\mathcal{P} \mathcal{T}_{X}$ (from left to right) according to the usual rule for composition of partial mappings, namely, that employed in the symmetric inverse monoid $\mathcal{I}_{X}$. Under this composition, $\mathcal{P} \mathcal{T}_{X}$ clearly forms a monoid, which we term the partial transformation monoid of $X$. Similarly, $\mathcal{P} \mathcal{T}_{X}^{*}$, the dual partial transformation monoid of $X$, is the collection of all partial mappings of $X$ with composition performed from right to left. We denote by $I_{A}$ the partial identity mapping on a subset $A \subseteq X$; the collection $E_{X}$ of all such partial identities forms a subsemilattice of both $\mathcal{P} \mathcal{T}_{X}$ and $\mathcal{P} \mathcal{T}_{X}^{*}$. We now consider the unary operation on partial transformations which is given by $\alpha \mapsto I_{\operatorname{dom} \alpha}$. In $\mathcal{P} \mathcal{T}_{X}$, we denote this operation by ${ }^{+}$; in $\mathcal{P} \mathcal{T}_{X}^{*}$, we denote it by ${ }^{*}$. Let $S$ be a semigroup. We call $S$ a two-sided restriction semigroup if

(1) $S$ is isomorphic to a subsemigroup of some $\mathcal{P} \mathcal{T}_{X}$ that is closed under ${ }^{+}$ (via an isomorphism $\phi$ );

(2) $S$ is isomorphic to a subsemigroup of some $\mathcal{P} \mathcal{T}_{Y}^{*}$ that is closed under * (via an isomorphism $\psi$ );

(3) the semilattices $\left\{(s \phi)^{+}: s \in S\right\}$ and $\left\{(s \psi)^{*}: s \in S\right\}$ are isomorphic.

Such semigroups have appeared in a range of contexts (see [10]) and have an alternative, abstract characterisation which will be used throughout this paper (see Section 2).

In extending the functions of Definitions 1.1 and 1.2 to the case of two-sided restriction semigroups, our particular interest is in obtaining a generalisation of the celebrated Ehresmann-Schein-Nambooripad Theorem (hereafter, ESN Theorem). This theorem establishes a fundamental connection between inverse semigroups and inductive groupoids. The formal statement of the ESN Theorem (as it appears in [16], for which book it provides the main focus) is as follows:

Theorem 1.3. [16, Theorem 4.1.8] The category of inverse semigroups and $(\vee, i)$ premorphisms is isomorphic to the category of inductive groupoids and ordered functors; the category of inverse semigroups and morphisms is isomorphic to the category of inductive groupoids and inductive functors.

(An ordered functor is simply an order-preserving functor, whilst a inductive functor is an ordered functor which also preserves the 'meet' operation in an inductive groupoid.) 
The 'morphisms' part of this result has already been extended to the case of two-sided restriction semigroups by Lawson [15]; in Lawson's result, inductive groupoids are replaced by inductive categories and morphisms by $(2,1,1)$ morphisms. We will complete the generalisation by considering the ' $(\vee, i)$-premorphisms' part. Furthermore, we will obtain a version of this theorem for $(\wedge, i)$ premorphisms, via the more general case of two-sided restriction semigroups; it is this type of premorphism which has proved most useful in recent years in the study of partial actions. We note that arbitrary $(\wedge, i)$-premorphisms do not compose to give another $(\wedge, i)$-premorphism. This problem is solved (both in the inverse case, and in the theory we will develop for restriction semigroups) if we also insist that the functions be order-preserving.

The structure of the paper is as follows. We begin with Section 2, in which we record some results on two-sided restriction semigroups which will be of use in later sections. Further preliminary definitions and results follow in Section 3 ; in the first part of the section, we introduce various category-theoretic notions, including that of an inductive category; in the second part, we define the Szendrei expansion of an inductive groupoid - a piece of algebraic machinery which will be needed briefly in Section [6. In Section 4 we define the notion of $(\vee, r)$ premorphisms for two-sided restriction semigroups and prove a new version (Theorem 4.1) of the ESN Theorem with these functions as the arrows of a category of two-sided restriction semigroups. We show that the existing result in the inverse case follows from ours as a corollary. In Section 5, we move onto the ' $\wedge$-premorphisms' part of the paper. By adapting concepts encountered in the study of partial actions, we prove yet another version (Theorem 5.1) of the ESN Theorem, this time for two-sided restriction semigroups and ordered $(\wedge, r)$ premorphisms. In fact, we will need to prove two versions of this theorem: it will turn out that the most naive notion of ' $(\wedge, r)$-premorphism', i.e., that obtained by replacing condition $(\wedge 2)^{\prime}$ in Definition 1.2 by a condition involving ${ }^{+}$and ${ }^{*}$, will not suffice for our purposes and we will therefore also need the notion of a strong $(\wedge, r)$-premorphism (see Theorem 5.9). A version of the ESN Theorem for inverse semigroups and ordered $(\wedge, i)$-premorphisms (Theorem 6.1) will follow in Section 6, as a corollary to the results of Section 5, thanks to our introduction of strong $(\wedge, r)$-premorphisms.

\section{Restriction SEMigroups}

In this section, we summarise the pertinent details of the theory of two-sided restriction semigroups. The material of this section appears in a range of published sources (see, for example, [4, 5, 8, 13, 15]). However, there are only two places where many of the relevant definitions and results have been collated into a single resource: the notes [9] and Chapter 2 of the author's Ph.D. thesis [11]. The reader is referred to these sources for further details and for more extensive 
references. For a more easily accessible published source, see Section 1 of [12], written for monoids.

Notice from our comments in the Introduction that we can easily define onesided versions of these semigroups: left restriction semigroups (subsemigroups of $\mathcal{P} \mathcal{T}_{X}$ closed under ${ }^{+}$) and right restriction semigroups (subsemigroups of $\mathcal{P} \mathcal{T}_{Y}^{*}$ closed under *). However, in this paper, we will only be interested in the two-sided version. From here on, we therefore drop the qualifier 'two-sided'; henceforth, whenever we use the term 'restriction semigroup', it can be taken to mean the twosided version. We also note here that left/right/two-sided restriction semigroups have appeared in a range of contexts under a number of different names: see [10] for further references. The term 'restriction semigroup' is a recent attempt to harmonise terminology and originates with [3].

In the Introduction, we saw the concrete characterisation of restriction semigroups as subsemigroups of partial transformation monoids. We now give an abstract description. Let $S$ be a semigroup and suppose that $E \subseteq E(S)$ is a subsemilattice of $S$. We define the (equivalence) relations $\widetilde{\mathcal{R}}_{E}$ and $\widetilde{\mathcal{L}}_{E}$ on $S$, with respect to $E$, by the rules that

$$
\begin{aligned}
& a \widetilde{\mathcal{R}}_{E} b \Longleftrightarrow \forall e \in E[e a=a \Leftrightarrow e b=b] ; \\
& a \widetilde{\mathcal{L}}_{E} b \Longleftrightarrow \forall e \in E[a e=a \Leftrightarrow b e=b],
\end{aligned}
$$

for $a, b \in S$. Thus, two elements $a, b$ are $\widetilde{\mathcal{R}}_{E}$-related if, and only if, they have the same left identities in $E$. Similarly, $a \widetilde{\mathcal{L}}_{E} b$ if, and only if, $a$ and $b$ have the same right identities in $E$. The abstract definition of a restriction semigroup runs as follows:

Definition 2.1. A semigroup $S$ with subsemilattice $E \subseteq E(S)$ is (two-sided) restriction semigroup (with respect to $E$ ) if

(1) every element $a$ is both $\widetilde{\mathcal{R}}_{E^{-}}$and $\widetilde{\mathcal{L}}_{E^{-}}$related to an idempotent in $E$, denoted $a^{+}$and $a^{*}$, respectively;

(2) $\widetilde{\mathcal{R}}_{E}$ is a left congruence, whilst $\widetilde{\mathcal{L}}_{E}$ is a right congruence;

(3) for all $a \in S$ and all $e \in E, a e=(a e)^{+} a$ and $e a=a(e a)^{*}$.

Thus, in a restriction semigroup $S$,

$$
a \widetilde{\mathcal{R}}_{E} b \Leftrightarrow a^{+}=b^{+} \quad \text { and } \quad a \widetilde{\mathcal{L}}_{E} \Leftrightarrow a^{*}=b^{*} .
$$

The idempotents $a^{+}$and $a^{*}$ are left and right identities for $a$, respectively. We note that $a^{+}$and $a^{*}$ are necessarily unique. It is also clear that if $e \in E$, then $e^{+}=e=e^{*}$.

If we were to consider only the parts of Definition 2.1 which relate to $\widetilde{\mathcal{R}}_{E}$ and ${ }^{+}$ (respectively, $\widetilde{\mathcal{L}}_{E}$ and ${ }^{*}$ ), then we would have a left (respectively, right) restriction semigroup. 
Using [9, Theorem 6.2], it is possible to connect the concrete and abstract approaches to restriction semigroups by showing that left (right) restriction semigroups are, up to isomorphism, precisely $(2,1)$-subalgebras of (dual) partial transformation monoids. However, two-sided restriction semigroups cannot be regarded as $(2,1,1)$-subalgebras of partial transformation monoids.

Note that $\mathcal{R} \subseteq \widetilde{\mathcal{R}}_{E}$ and $\mathcal{L} \subseteq \widetilde{\mathcal{L}}_{E}$, for any $E$. It is easy to see that, in a regular semigroup, $\mathcal{R}=\widetilde{\mathcal{R}}_{E(S)}$ and $\mathcal{L}=\widetilde{\mathcal{L}}_{E(S)}$. It follows that restriction semigroups generalise inverse semigroups, since every inverse semigroup is a restriction semigroup with $a^{+}=a a^{-1}$ and $a^{*}=a^{-1} a$. Restriction semigroups also generalise the ample (formerly, type-A) semigroups of Fountain [4, 5].

We note a pair of useful identities which follow easily from condition (2) of Definition 2.1:

Lemma 2.2. [5, Proposition 1.6(2)] Let $S$ be a restriction semigroup, for some $E \subseteq E(S)$, and let $s, t \in S$. Then $(s t)^{+}=\left(s t^{+}\right)^{+}$and $(s t)^{*}=\left(s^{*} t\right)^{*}$.

Both $\mathcal{P} \mathcal{T}_{X}$ and $\mathcal{P} \mathcal{T}_{X}^{*}$ possess an obvious natural partial order (i.e., a partial order which is compatible with multiplication and which restricts to the usual partial order on idempotents), defined by

$$
\alpha \leq \beta \Longleftrightarrow \alpha=\left.\beta\right|_{\text {dom } \alpha}
$$

In the abstract characterisation of a restriction semigroup $S$, the ordering of (2.1) becomes the following natural partial order

$$
a \leq b \Longleftrightarrow a=e b \Longleftrightarrow a=b f,
$$

for some idempotents $e, f \in E$. Equivalently,

$$
a \leq b \Longleftrightarrow a=a^{+} b \Longleftrightarrow a=b a^{*} .
$$

This equivalence is justified (for the ${ }^{~}{ }^{\prime}$, part) in [12, $\left.\S 1\right]$.

\section{FURTHER PRELIMINARIES}

In this section, we describe the relevant existing results connecting restriction semigroups and inductive categories, and introduce some algebraic machinery for later use.

3.1. Categories. We begin by giving an explicit definition of an arbitrary category. The results quoted in this subsection will take care of the 'objects' parts of our main results: the upcoming ESN-type Theorems 4.1, 5.1, 5.9 and 6.1

Let $C$ be a class and let - be a partial binary operation on $C$. For $x, y \in C$, we will write ' $\exists x \cdot y$ ' to mean 'the product $x \cdot y$ is defined'. Whenever we write $' \exists(x \cdot y) \cdot z^{\prime}$, it will be understood that we mean $\exists x \cdot y$ and $\exists(x \cdot y) \cdot z$. An element $e \in C$ is idempotent if $\exists e \cdot e$ and $e \cdot e=e$. The identities (or objects) of $C$ are those idempotents $e$ which satisfy: $[\exists e \cdot x \Rightarrow e \cdot x=x]$ and $[\exists x \cdot e \Rightarrow x \cdot e=x]$. We denote the subset of identities of $C$ by $C_{o}$ (' $o$ ' for 'objects'). 
Definition 3.1. Let $C$ be a class and let - be a partial binary operation on $C$. The pair $(C, \cdot)$ is a category if the following conditions hold:

(Ca1) $\exists x \cdot(y \cdot z) \Longleftrightarrow \exists(x \cdot y) \cdot z$, in which case $x \cdot(y \cdot z)=(x \cdot y) \cdot z$;

(Ca2) $\exists x \cdot(y \cdot z) \Longleftrightarrow \exists x \cdot y$ and $\exists y \cdot z$;

(Ca3) for each $x \in C$, there exist unique identities $\mathbf{d}(x), \mathbf{r}(x) \in C_{o}$ such that $\exists \mathbf{d}(x) \cdot x$ and $\exists x \cdot \mathbf{r}(x)$.

The identity $\mathbf{d}(x)$ is called the domain of $x$ and $\mathbf{r}(x)$ is the range of $x$. If $C$ is simply a set, then we call $(C, \cdot)$ a small category. A groupoid is a small category in which the following additional condition holds:

(G) for each $x \in G$, there is an $x^{-1} \in G$ such that $\exists x \cdot x^{-1}$ and $\exists x^{-1} \cdot x$, with $x \cdot x^{-1}=\mathbf{d}(x)$ and $x^{-1} \cdot x=\mathbf{r}(x)$.

This is essentially the definition of [16, p. 78], but with domain and range switched, since we will be composing functions from left to right. We note that if $(C, \cdot)$ is a category and $x, y \in C$, then

$$
\exists x \cdot y \Longleftrightarrow \mathbf{r}(x)=\mathbf{d}(y) .
$$

Note further that if $(C, \cdot)$ has precisely one object $e$, then all products are necessarily defined and it follows that $(C, \cdot)$ is, in fact, a monoid with identity $e$. Thus, a category may be regarded as a generalisation of a monoid.

Before proceeding further, we make the important observation that in the remainder of this paper, the word 'category' will be used in two slightly different, though equivalent, senses. First, we will have 'categories' as generalised monoids, in the sense of Definition 3.1, second, we will have 'categories' in the more traditional 'objects' and 'morphisms' sense (as in, for example, [14, Definition 1.1]). All categories considered in the first sense will be small categories. Thus, for example, the 'inductive categories' of Theorem 4.1 are (small) categories in the sense of Definition 3.1, but the category of inductive categories (and that of restriction semigroups) is regarded as a category in the traditional 'objects' and 'morphisms' sense.

We now introduce an ordering on a category $C$ :

Definition 3.2. Let $(C, \cdot)$ be a category (in the sense of Definition 3.1) and let $C$ be partially ordered by $\leq$. The triple $(C, \cdot, \leq)$ is an ordered category if the following conditions hold:

(Or1) $a \leq c, b \leq d, \exists a \cdot b$ and $\exists c \cdot d \Longrightarrow a \cdot b \leq c \cdot d$;

(Or2) $a \leq b \Longrightarrow \mathbf{r}(a) \leq \mathbf{r}(b)$ and $\mathbf{d}(a) \leq \mathbf{d}(b)$;

(Or3) (i) $f \in C_{o}, a \in C, f \leq \mathbf{r}(a) \Longrightarrow$ there exists a unique element, denoted $a \mid f$, such that $a \mid f \leq a$ and $\mathbf{r}(a \mid f)=f$;

(ii) $f \in C_{o}, a \in C, f \leq \mathbf{d}(a) \Longrightarrow$ there exists a unique element, denoted $f \mid a$, such that $f \mid a \leq a$ and $\mathbf{d}(f \mid a)=f$.

An ordered groupoid is a small ordered category in which condition (G) holds. 
The element $a \mid f$ of condition (Or3)(i) is called the corestriction (of $a$ to $f$ ), whilst the element $f \mid a$ of condition (Or3)(ii) is called the restriction (of $f$ to a). We note that, for $e, f \in C_{o}$, there is no ambiguity in the notation ' $e \mid f$ ': the restriction $e \mid f$ and the corestriction $e \mid f$ coincide whenever both are defined. Note further that in this case we have $e=f=e \mid f$, by definition of restrictions and corestrictions. It is clear that any function which respects domains, ranges and ordering (for example, an ordered functor) will also respect restrictions and corestrictions.

We record the following properties of the restriction and corestriction in an ordered category:

Lemma 3.3. Let $(C, \cdot, \leq)$ be an ordered category. Let $a \in C$ and $e, f \in C_{o}$ with $f \leq e \leq \mathbf{r}(a)$. Then $(a \mid e)|f=a| f$. Consequently, $a|f \leq a| e$.

Dually, if $a \in C$ and $e, f \in C_{o}$ with $f \leq e \leq \mathbf{d}(a)$, then $f|(e \mid a)=f| a$, hence $f|a \leq e| a$.

(Our Lemma 3.3 is essentially Lemma 4.4 of [15].)

Note that in an inductive groupoid $G$, corestrictions may be defined in terms of restrictions: for $a \in G$ and $f \in G_{o}$ with $f \leq \mathbf{r}(a)$, the corestriction $a \mid f$ is given by $a \mid f=\left(f \mid a^{-1}\right)^{-1}$ [7, p. 178].

Lemma 3.4. Let $(C, \cdot, \leq)$ be an ordered category and let $a, b \in C$. If $a \leq b$, then $a=\mathbf{d}(a)|b=a| \mathbf{r}(b)$. In particular, $a=\mathbf{d}(a)|a=a| \mathbf{r}(a)$.

(This result appears in [1] for inductive cancellative categories; the proof carries over the present case without modification.)

In an ordered category $(C, \cdot, \leq)$, if the greatest lower bound of $e, f \in C_{o}$ exists, then we denote it by $e \wedge f$.

Definition 3.5. An inductive category $(C, \cdot, \leq)$ is an ordered category in which the following additional condition holds:

(I) $e, f \in C_{o} \Longrightarrow e \wedge f$ exists in $C_{o}$.

An inductive groupoid is a small inductive category in which condition $(G)$ holds.

The foregoing sequence of definitions has been building to the following result, which appears in [15, §5]:

Theorem 3.6. Let $S$ be a restriction semigroup with respect to some semilattice $E$, and let $S$ have natural partial order $\leq$. If we define the restricted product . in $S$ by

$$
a \cdot b= \begin{cases}a b & \text { if } a^{*}=b^{+} ; \\ \text {undefined } & \text { otherwise, }\end{cases}
$$

then $(S, \cdot, \leq)$ is an inductive category with $S_{o}=E, \mathbf{d}(x)=x^{+}$and $\mathbf{r}(x)=x^{*}$.

The restriction $f \mid a$ in the inductive category $(S, \cdot \leq)$ is simply the product $f a$ in the restriction semigroup $S$, since $f a \leq a$ and $(f a)^{+}=\left(f a^{+}\right)^{+}=f$, by Lemma 2.2, as $f \leq a^{+}$. Similarly, $a \mid f=a f$ and $e \wedge f=e f$. 
We now define the pseudoproduct $\otimes$ in an inductive category $(C, \cdot, \leq)$ by 2

$$
a \otimes b=[a \mid \mathbf{r}(a) \wedge \mathbf{d}(b)] \cdot[\mathbf{r}(a) \wedge \mathbf{d}(b) \mid b] .
$$

The pseudoproduct is everywhere-defined in $C$ (thanks to (I)) and coincides with the product · in $C$ whenever - is defined. To see this, recall that if $\exists a \cdot b$, then $\mathbf{r}(a)=\mathbf{d}(b)$, so that $a \otimes b=[a \mid \mathbf{r}(a)] \cdot[\mathbf{d}(b) \mid b]$. Then $a \otimes b=a \cdot b$, by Lemma 3.4.

We record the following properties of the pseudoproduct for later use:

Lemma 3.7. Let $(C, \cdot, \leq)$ be an inductive category and let $a \in C, e \in C_{o}$. Then $e \otimes a=e \wedge \mathbf{d}(a) \mid a \quad$ and $\quad a \otimes e=a \mid \mathbf{r}(a) \wedge e$.

(This result appears in [1] for inductive cancellative categories; the proof carries over the present case without modification.)

Theorem 3.8. [15, §5] If $(C, \cdot, \leq)$ is an inductive category, then $(C, \otimes)$ is a restriction semigroup with respect to $C_{o}$.

Let $S$ be a restriction semigroup. We will denote the inductive category associated to $S$ by $\mathbf{C}(S)$. Similarly, if $C$ is an inductive category, then we will denote its associated restriction semigroup by $\mathbf{S}(C)$. The following result is implicit in [15, §5]:

Theorem 3.9. Let $S$ be a restriction semigroup and $C$ be an inductive category. Then $\mathbf{S}(\mathbf{C}(S))=S$ and $\mathbf{C}(\mathbf{S}(C))=C$.

Theorems 3.6, 3.8 and 3.9 prove the equivalence of the objects of the categories in the upcoming Theorems 4.1, 5.1 and 5.9, It only remains for us to deal with the arrows.

We conclude this subsection with a property of ordered categories which will be immensely useful later in the paper.

Lemma 3.10. Let $(C, \cdot, \leq)$ be an ordered category and let $a, b \in C$. If $a, b \leq c$, for some $c \in C$, and either $\mathbf{d}(a)=\mathbf{d}(b)$ or $\mathbf{r}(a)=\mathbf{r}(b)$, then $a=b$.

(This result appears in [1] for inductive cancellative categories; the proof carries over the present case without modification.)

3.2. The Szendrei expansion of an inductive groupoid. We take this opportunity to introduce an algebraic tool which we will require briefly in Section 6 : the Szendrei expansion of an inductive groupoid, as defined by Gilbert [7].

The concept of a 'semigroup expansion' was first introduced by Birget and Rhodes in [2], and is simply a special type of functor from one category of semigroups to another. Amongst the various expansions in the literature are a number of different versions of the so-called Szendrei expansion. The original Szendrei

\footnotetext{
${ }^{2}$ In the interests of reducing the number of brackets, expressions such as $a \mid \mathbf{r}(a) \wedge \mathbf{d}(b)$ will be understood to mean $a \mid(\mathbf{r}(a) \wedge \mathbf{d}(b))$; of course, the alternative, $(a \mid \mathbf{r}(a)) \wedge \mathbf{d}(b)$, makes no sense if $a$ is not an identity.
} 
expansion was introduced in [18] and was there applied to groups; it was subsequently extended to monoids in [6].

Gilbert [7] has studied the partial actions of inductive groupoids as a way of informing the study of partial actions of inverse semigroups. In the course of this work, he has defined a Szendrei expansion for inductive groupoids. Let $G$ be an inductive groupoid. For each identity $e \in G_{o}$, we define the $\operatorname{set} \operatorname{star}_{e}(G)$ by

$$
\operatorname{star}_{e}(G)=\{g \in G: \mathbf{d}(g)=e\} .
$$

Let $\mathscr{F}_{e}\left(\operatorname{star}_{e}(G)\right)$ be the collection of all finite subsets of $\operatorname{star}_{e}(G)$ which contain $e$, and put

$$
\mathscr{F}_{*}(G)=\bigcup_{e \in G_{o}} \mathscr{F}_{e}\left(\operatorname{star}_{e}(G)\right)
$$

We now make the following definition:

Definition 3.11. [7, p. 179] Let $G$ be an inductive groupoid. The Szendrei expansion $3^{3}$ of $G$ is the set

$$
\mathrm{Sz}(G)=\left\{(U, u) \in \mathscr{F}_{*}(G) \times G: u \in U\right\},
$$

together with the operation

$$
(U, u)(V, v)= \begin{cases}(U, u v) & \text { if } \mathbf{r}(u)=\mathbf{d}(v) \text { and } U=u V \\ \text { undefined } & \text { otherwise. }\end{cases}
$$

We note that $\mathrm{Sz}(G)$ has identities

$$
\mathrm{Sz}(G)_{o}=\left\{(E, e) \in \mathrm{Sz}(G): e \in G_{o}\right\} .
$$

We note also that Gilbert's definition applies, more generally, to ordered groupoids, but we will only concern ourselves with the inductive case.

Proposition 3.12. [7, Proposition 3.1 \& Corollary 3.3] If $G$ is an inductive groupoid, then $\mathrm{Sz}(G)$ is an inductive groupoid with $(U, u)^{-1}=\left(u^{-1} U, u^{-1}\right)$,

$$
\mathbf{d}((U, u))=(U, \mathbf{d}(u)), \quad \mathbf{r}((U, u))=\left(u^{-1} U, \mathbf{r}(u)\right),
$$

and ordering

$$
(U, u) \leq(V, v) \Longleftrightarrow u \leq v \text { in } G \text { and } \mathbf{d}(u) \mid V \subseteq U,
$$

where $\mathbf{d}(u) \mid V=\{\mathbf{d}(u) \mid w: w \in V\}$. For $(E, e) \in \operatorname{Sz}(G)_{o}$ with $(E, e) \leq \mathbf{d}((A, a))$, the restriction $\sqrt{4}$ is given by

$$
(E, e) \mid(A, a)=(E, e \mid u)
$$

whilst, for any $(E, e),(F, f) \in \mathrm{Sz}(G)_{o}$, we have

$$
(E, e) \wedge(F, f)=((e \wedge f) \mid(E \cup F), e \wedge f) .
$$

\footnotetext{
${ }^{3}$ Gilbert refers to this as the Birget-Rhodes expansion but we adopt the term Szendrei expansion for consistency with previous definitions.

${ }^{4}$ We need not give the corestriction explicitly, thanks to the comments following Lemma 3.3.
} 
The pseudoproduct in $\operatorname{Sz}(G)$ may be written:

$$
(U, u) \otimes(V, v)=(\mathbf{d}(u \mid \mathbf{r}(u) \wedge \mathbf{d}(v)) \mid U \cup u \otimes V, u \otimes v),
$$

where $u \otimes V=\{u \otimes w: w \in V\}$ [7, Theorem 3.2]. Note also that we can inject any inductive groupoid $G$ into $\operatorname{Sz}(G)$ via the mapping $\iota: G \rightarrow \operatorname{Sz}(G)$, given by $g \iota=(\{\mathbf{d}(g), g\}, g)$. The Szendrei expansion of an inductive groupoid will be used to prove Lemma 6.5.

\section{4. $(\vee, r)$-PREMORPHISMS}

The goal of this section is the proof of the following theorem, and its connection with the ' $(\vee, i)$-premorphisms' part of Theorem 1.3 .

Theorem 4.1. The category of restriction semigroups and $(\vee, r)$-premorphisms is isomorphic to the category of inductive categories and ordered functors.

We note that the 'objects' part of this theorem has been taken care of by the results of Section 3, it remains to deal with the 'arrows' part. We begin by defining the notion of a $(\vee, r)$-premorphism.

Definition 4.2. Let $S$ and $T$ be restriction semigroups. A ( $\vee, r)$-premorphism is a function $\theta: S \rightarrow T$ such that

$(\vee 1)(s t) \theta \leq(s \theta)(t \theta)$;

$(\vee 2) s^{+} \theta \leq(s \theta)^{+}$and $s^{*} \theta \leq(s \theta)^{*}$.

The notion of a $(\vee, r)$-premorphism generalises that of a $(\vee, i)$-premorphism. To see this, we must first record the following concerning $(\vee, i)$-premorphisms:

Lemma 4.3. [16, Theorem 3.1.5] Let $\theta: S \rightarrow T$ be a $(\vee, i)$-premorphism. Then $\theta$ respects inverses and the natural partial order.

We now have:

Lemma 4.4. Let $\theta: S \rightarrow T$ be a function between inverse semigroups. Then $\theta$ is a $(\vee, i)$-premorphism if, and only if, it is a $(\vee, r)$-premorphism.

Proof. $(\Leftarrow)$ Immediate.

$(\Rightarrow)$ Suppose that $\theta: S \rightarrow T$ is a $(\vee, i)$-premorphism. Then

$$
s^{+} \theta=\left(s s^{-1}\right) \theta \leq(s \theta)\left(s^{-1} \theta\right)=(s \theta)(s \theta)^{-1}=(s \theta)^{+},
$$

using Lemma 4.3. Similarly, $s^{*} \theta \leq(s \theta)^{*}$.

We note some useful properties of $(\vee, r)$-premorphisms:

Lemma 4.5. Let $S$ and $T$ be restriction semigroups with respect to semilattices $E$ and $F$, respectively. If $\theta: S \rightarrow T$ is a $(\vee, r)$-premorphism, then

(a) $e \in E(S) \Rightarrow e \theta \in E(T)$;

(b) $e \in E \Rightarrow e \theta \in F$;

(c) $(s \theta)^{+}=s^{+} \theta$ and $(s \theta)^{*}=s^{*} \theta$; 
(d) $\theta$ is order-preserving.

Proof. (a) Let $e \in E(S)$. From (V1), $e \theta=e^{2} \theta \leq(e \theta)^{2}$. Then, by (2.3) $), e \theta=$ $(e \theta)^{+}(e \theta)^{2}=(e \theta)^{2}$, hence $e \theta \in E(T)$.

(b) Let $e \in E$. From $(\vee 2), e \theta=e^{+} \theta \leq(e \theta)^{+}$. Again by (2.3), $e \theta=$ $(e \theta)^{+}(e \theta)^{+}=(e \theta)^{+}$, hence $e \theta \in F$.

(c) We deal with the "+' part; the ${ }^{(*)}$ part is similar. We will show that $(s \theta)^{+} \leq$ $s^{+} \theta$. The desired result will then follow by combining this with ( $\left.\vee 2\right)$. Let $s \in S$. From $(\vee 1), s \theta=\left(s^{+} s\right) \theta \leq\left(s^{+} \theta\right)(s \theta)$, so

$$
s \theta=\left(s^{+} \theta\right)(s \theta)(s \theta)^{*}=\left(s^{+} \theta\right)(s \theta),
$$

once again by (2.3). Applying + to both sides of (4.1) gives

$$
(s \theta)^{+}=\left(\left(s^{+} \theta\right)(s \theta)\right)^{+}=\left(\left(s^{+} \theta\right)(s \theta)^{+}\right)^{+}=\left(s^{+} \theta\right)(s \theta)^{+},
$$

using Lemma 2.2, and since $s^{+} \theta \in F$, by (b). Hence $(s \theta)^{+} \leq s^{+} \theta$.

(d) Suppose that $s \leq t$ in $S$. Then $s=s^{+} t$, by (2.3), so $s \theta=\left(s^{+} t\right) \theta \leq$ $\left(s^{+} \theta\right)(t \theta) \leq t \theta$ in $T$.

Using Lemma 4.5(d), it is easily verified that the composition of two $(\mathrm{V}, r)$ premorphisms is a $(\vee, r)$-premorphism, hence restriction semigroups and $(\vee, r)$ premorphisms constitute a category. We are now ready to prove the 'arrows' part of Theorem 4.1, which we will break down into two parts (Propositions 4.8 and 4.9). Before we do so, however, we first record the following, which will be used a number of times in the remainder of this paper:

Lemma 4.6. Let $\alpha: S \rightarrow T$ be an order-preserving function of restriction semigroups. We define $\mathbf{C}(\alpha): \mathbf{C}(S) \rightarrow \mathbf{C}(T)$ to be the same function on the underlying sets. Then $\mathbf{C}(\alpha)$ is order-preserving.

Let $\beta: C \rightarrow D$ be an order-preserving function of inductive categories. We define $\mathbf{S}(\beta): \mathbf{S}(C) \rightarrow \mathbf{S}(D)$ to be the same function on the underlying sets. Then $\mathbf{S}(\beta)$ is order-preserving.

Proof. Let $s, t \in \mathbf{C}(S)$. Then

$$
s \leq t \text { in } \mathbf{C}(S) \Rightarrow s \leq t \text { in } S \Rightarrow s \alpha \leq t \alpha \text { in } T \Rightarrow s \mathbf{C}(\alpha) \leq t \mathbf{C}(\alpha) \text { in } \mathbf{C}(T) .
$$

The proof of the second part is similar.

We now also give a formal definition for the ordered functors which appear in Theorem 4.1;

Definition 4.7. Let $\phi: C \rightarrow D$ be a function between ordered categories $C$ and $D$. We call $\phi$ an ordered functor if

(1) $\exists x \cdot y$ in $C \Rightarrow \exists(x \phi) \cdot(y \phi)$ in $D$ and $(x \phi) \cdot(y \phi)=(x \cdot y) \phi$;

(2) $x \leq y$ in $C \Rightarrow x \phi \leq y \phi$ in $D$. 
Proposition 4.8. Let $S$ and $T$ be restriction semigroups with respect to semilattices $E$ and $F$, respectively. Let $\theta: S \rightarrow T$ be a $(\vee, r)$-premorphism. We define $\Theta:=\mathbf{C}(\theta): \mathbf{C}(S) \rightarrow \mathbf{C}(T)$ to be the same function on the underlying sets. Then $\Theta$ is an ordered functor with respect to the restricted products in $\mathbf{C}(S)$ and $\mathbf{C}(T)$.

Proof. Since $\theta$ maps $E$ into $F$, plus $\mathbf{C}(S)_{o}=E$ and $\mathbf{C}(T)_{o}=F$, we see that $\Theta$ maps identities in $\mathbf{C}(S)$ to identities in $\mathbf{C}(T)$.

We now show that $\Theta$ respects restricted products. Suppose that $\exists s \cdot t$ in $\mathbf{C}(S)$. Then $s^{*}=t^{+}$in $S$, hence $(s \Theta)^{*}=(s \theta)^{*}=s^{*} \theta=t^{+} \theta=(t \theta)^{+}=(t \Theta)^{+}$, by Lemma 4.5(c). We conclude that $\exists(s \Theta) \cdot(t \Theta)$ in $\mathbf{C}(T)$. We will now use Lemma 3.10 to show that $(s \cdot t) \Theta=(s \Theta) \cdot(t \Theta)$. Note first of all that $(s \cdot t) \Theta$ $=(s t) \theta \leq(s \theta)(t \theta)$ and that $(s \Theta) \cdot(t \Theta)=(s \theta)(t \theta) \leq(s \theta)(t \theta)$. Now, using Lemma 2.2 ,

$$
\mathbf{r}((s \cdot t) \Theta)=(s t) \theta^{*}=(s t)^{*} \theta=\left(s^{*} t\right)^{*} \theta=t^{*} \theta,
$$

since $s^{*}=t^{+}$. Also,

$$
\begin{aligned}
\mathbf{r}((s \Theta) \cdot(t \Theta)) & =((s \theta)(t \theta))^{*}=\left((s \theta)^{*}(t \theta)\right)^{*}=\left(\left(s^{*} \theta\right)(t \theta)\right)^{*}=\left(\left(t^{+} \theta\right)(t \theta)\right)^{*} \\
& =\left((t \theta)^{+}(t \theta)\right)^{*}=(t \theta)^{*}=t^{*} \theta=\mathbf{r}((s \cdot t) \Theta) .
\end{aligned}
$$

Hence, by Lemma 3.10, $(s \cdot t) \Theta=(s \Theta) \cdot(t \Theta)$.

Finally, $\Theta$ is order-preserving, by Lemma 4.6.

Proposition 4.9. Let $\phi: C \rightarrow D$ be an ordered functor of inductive categories. We define $\Phi:=\mathbf{S}(\phi): \mathbf{S}(C) \rightarrow \mathbf{S}(D)$ to be the same function on the underlying sets. Then $\Phi$ is a $(\vee, r)$-premorphism with respect to the pseudoproducts in $\mathbf{S}(C)$ and $\mathbf{S}(D)$.

Proof. Let $s, t \in \mathbf{S}(C)$. Note that if $e=s^{*} \otimes t^{+}$, then

$$
\begin{aligned}
(s \otimes e)^{*} & =\left(s \otimes s^{*} \otimes t^{+}\right)^{*}=\left(s \otimes t^{+}\right)^{*}=\left(s^{*} \otimes t^{+}\right)^{*}=s^{*} \otimes t^{+} \\
& =\left(s^{*} \otimes t^{+}\right)^{+}=\left(s^{*} \otimes t\right)^{+}=\left(s^{*} \otimes t^{+} \otimes t\right)^{+}=(e \otimes t)^{+},
\end{aligned}
$$

so $\exists(s \otimes e) \cdot(e \otimes t)$ and $(s \otimes e) \cdot(e \otimes t)=s \otimes t$. Since $\phi$ is a functor, we have $(s \otimes t) \phi=(s \otimes e) \phi \cdot(e \otimes t) \phi=(s \otimes e) \phi \otimes(e \otimes t) \phi$, using the fact that $\cdot$ and $\otimes$ coincide whenever $\cdot$ is defined. Now, $s \otimes e \leq s$ and $e \otimes t \leq t$, so $(s \otimes e) \phi \leq s \phi$ and $(e \otimes t) \phi \leq t \phi$. Hence

$$
(s \otimes t) \Phi=(s \otimes t) \phi=(s \otimes e) \phi \otimes(e \otimes t) \phi \leq(s \phi) \otimes(t \phi)=(s \Phi) \otimes(t \Phi) .
$$

Let $s \in S$. Since functors preserve domains, we have $(s \Phi)^{+}=\mathbf{d}(s \phi)=\mathbf{d}(s) \phi=$ $s^{+} \Phi$. Then, in particular, $(s \Phi)^{+} \geq s^{+} \Phi$. Similarly, $(s \Phi)^{*} \geq s^{*} \Phi$.

It is clear that if $\theta: S \rightarrow T$ is a $(\vee, r)$-premorphism and $\phi: C \rightarrow D$ is an ordered functor, then $\mathbf{S}(\mathbf{C}(\theta))=\theta$ and $\mathbf{C}(\mathbf{S}(\phi))=\phi$. Furthermore, if $\theta^{\prime}: T \rightarrow T^{\prime}$ is another $(\vee, r)$-premorphism of restriction semigroups, and $\phi^{\prime}: D \rightarrow D^{\prime}$ is another ordered functor of inductive categories, then $\mathbf{C}\left(\theta \theta^{\prime}\right)=\mathbf{C}(\theta) \mathbf{C}\left(\theta^{\prime}\right)$ and $\mathbf{S}\left(\phi \phi^{\prime}\right)=\mathbf{S}(\phi) \mathbf{S}\left(\phi^{\prime}\right)$. We have therefore proved Theorem 4.1. 
The constructions of Theorems 3.6 and 3.8 carry over to the inverse case in such a way that if $S$ is an inverse semigroup and $G$ is an inductive groupoid, then $\mathbf{C}(S)$ is an inductive groupoid and $\mathbf{S}(G)$ is an inverse semigroup (see [16]). Then, using Lemma 4.4, we see that the ' $\vee$-premorphisms' part of Theorem 1.3 follows from Theorem 4.1 as a corollary.

\section{5. $(\wedge, r)$-PREMORPHISMS}

We now turn our attention to the derivation of an ESN-type Theorem for ' $\wedge$ premorphisms'. Two results of this type will be proved in this section. The first will employ the more naive version of a ' $(\wedge, r)$-premorphism', alluded to in the Introduction. However, we will not be able to prove an analogue of Lemma 4.4 for these $(\wedge, r)$-premorphisms. In order to make the desired connection with the functions of Definition 1.2, and thereby deduce an ESN-type theorem for inverse semigroups and $(\wedge, i)$-premorphisms, we need the notion of a strong $(\wedge, r)$ premorphism. In the second part of this section, we will prove an ESN-type theorem for strong $(\wedge, r)$-premorphisms which will follow from the weaker version as a corollary.

5.1. The weaker case: ordered $(\wedge, r)$-premorphisms. The goal of this subsection is the proof of the following theorem:

Theorem 5.1. The category of restriction semigroups and ordered $(\wedge, r)$-premorphisms is isomorphic to the category of inductive categories and inductive category prefunctors.

We note that the 'objects' part of this theorem has been taken care of by the results of Section 3, it remains to deal with the 'arrows' part.

We make the following definition, based upon the one-sided case in [10]:

Definition 5.2. A function $\theta: S \rightarrow T$ between restriction semigroups is called a $(\wedge, r)$-premorphism if

$(\wedge 1)(s \theta)(t \theta) \leq(s t) \theta$

$(\wedge 2)(s \theta)^{+} \leq s^{+} \theta$ and $(s \theta)^{*} \leq s^{*} \theta$.

If, in addition, $\theta$ is order-preserving, we call it an ordered $(\wedge, r)$-premorphism.

We note that whilst a $(\vee, r)$-premorphism is automatically order-preserving, this is not the case for a $(\wedge, r)$-premorphism: we must demand this explicitly. As noted in the Introduction, arbitrary $(\wedge, r)$-premorphisms do not compose to give a $(\wedge, r)$-premorphism. However, it is easily verified that the composition of two ordered $(\wedge, r)$-premorphisms is an ordered $(\wedge, r)$-premorphism. Restriction semigroups together with ordered $(\wedge, r)$-premorphisms therefore form a category.

Lemma 5.3. Let $S$ and $T$ be restriction semigroups with respect to semilattices $E$ and $F$, respectively. Let $\theta: S \rightarrow T$ be an ordered $(\wedge, r)$-premorphism. If $e \in E$, then $e \theta \in F$. 
Proof. Let $e \in E$. Then, by $(\wedge 2),(e \theta)^{+} \leq e^{+} \theta=e \theta$. By (2.3), we have $(e \theta)^{+}=$ $(e \theta)^{+}(e \theta)=e \theta \in F$.

We must now define a corresponding function between inductive categories:

Definition 5.4. A function $\psi: C \rightarrow D$ between inductive categories is called an inductive category prefunctor if

(ICP1) $\exists s \cdot t$ in $C \Rightarrow(s \psi) \otimes(t \psi) \leq(s \cdot t) \psi$;

(ICP2) $\mathbf{d}(s \psi) \leq \mathbf{d}(s) \psi$ and $\mathbf{r}(s \psi) \leq \mathbf{r}(s) \psi$;

(ICP3) $s \leq t$ in $C \Rightarrow s \psi \leq t \psi$ in $D$;

(ICP4) (a) for $a \in C$ and $f \in C_{o}, a \psi \mid \mathbf{r}(a \psi) \wedge f \psi \leq(a \mid \mathbf{r}(a) \wedge f) \psi$;

(b) for $a \in C$ and $e \in C_{o}, e \psi \wedge \mathbf{d}(a \psi) \mid a \psi \leq(e \wedge \mathbf{d}(a) \mid a) \psi$.

Note that we can use Lemma 3.7 to rewrite condition (ICP4) in a more compact form:

(ICP4)' (a) for $a \in C$ and $f \in C_{o}, a \psi \otimes f \psi \leq(a \otimes f) \psi$;

(b) for $a \in C$ and $e \in C_{o}, e \psi \otimes a \psi \leq(e \otimes a) \psi$.

Lemma 5.5. Let $\psi: C \rightarrow D$ be an inductive category prefunctor of inductive categories. If $e \in C_{o}$, then $e \psi \in D_{o}$.

Proof. Let $e \in C_{o}$, so that $e=e^{+}$in $\mathbf{S}(C)$. By (ICP2), $\mathbf{d}(e \psi) \leq \mathbf{d}(e) \psi$ in $D$, so $(e \psi)^{+} \leq e^{+} \psi=e \psi$ in $\mathbf{S}(D)$. Then, by definition of ordering in $\mathbf{S}(D)$, $(e \psi)^{+}=(e \psi)^{+} \otimes(e \psi)=e \psi$. Thus $\mathbf{d}(e \psi)=e \psi$ in $D$. Hence $e \psi \in D_{o}$.

Lemma 5.6. The composition of two inductive category prefunctors is an inductive category prefunctor.

Proof. Let $\psi_{1}: U \rightarrow V$ and $\psi_{2}: V \rightarrow W$ be inductive category prefunctors of inductive categories $U, V$ and $W$. It is easy to show that $\psi_{1} \psi_{2}$ satifies (ICP2)(ICP4); (ICP1), however, is a little trickier. Let $s, t \in U$ and suppose that $\exists s \cdot t$, i.e., $\mathbf{r}(s)=\mathbf{d}(t)$. Put $x=s \psi_{1}$ and $y=t \psi_{1}$. Then

$$
\begin{aligned}
\left(s \psi_{1} \psi_{2}\right) \otimes\left(t \psi_{1} \psi_{2}\right) & =\left(x \psi_{2}\right) \otimes\left(y \psi_{2}\right) \\
& =\left(x \psi_{2} \mid \mathbf{r}\left(x \psi_{2}\right) \wedge \mathbf{d}\left(y \psi_{2}\right)\right) \cdot\left(\mathbf{r}\left(x \psi_{2}\right) \wedge \mathbf{d}\left(y \psi_{2}\right) \mid y \psi_{2}\right) \\
& =\left(x \psi_{2} \mid \mathbf{r}\left(x \psi_{2}\right) \wedge \mathbf{d}\left(y \psi_{2}\right)\right) \otimes\left(\mathbf{r}\left(x \psi_{2}\right) \wedge \mathbf{d}\left(y \psi_{2}\right) \mid y \psi_{2}\right)
\end{aligned}
$$

( since $\cdot$ and $\otimes$ coincide whenever $\cdot$ is defined)

$$
\leq\left(x \psi_{2} \mid \mathbf{r}\left(x \psi_{2}\right) \wedge \mathbf{d}(y) \psi_{2}\right) \otimes\left(\mathbf{r}(x) \psi_{2} \wedge \mathbf{d}\left(y \psi_{2}\right) \mid y \psi_{2}\right)
$$

(by Lemma [3.3, since $\mathbf{r}\left(x \psi_{2}\right) \wedge \mathbf{d}\left(y \psi_{2}\right) \leq \mathbf{r}\left(x \psi_{2}\right) \wedge \mathbf{d}(y) \psi_{2}$, etc.)

$$
\begin{aligned}
& \leq(x \mid \mathbf{r}(x) \wedge \mathbf{d}(y)) \psi_{2} \otimes(\mathbf{r}(x) \wedge \mathbf{d}(y) \mid y) \psi_{2}, \text { by ICP4 } \\
& \leq((x \mid \mathbf{r}(x) \wedge \mathbf{d}(y)) \cdot(\mathbf{r}(x) \wedge \mathbf{d}(y) \mid y)) \psi_{2}, \text { by ICP1 } \\
& =(x \otimes y) \psi_{2} .
\end{aligned}
$$


But $x \otimes y=\left(s \psi_{1}\right) \otimes\left(t \psi_{1}\right) \leq(s \cdot t) \psi_{1}$, by (ICP1). Then, by (ICP3) for $\psi_{2}$, $\left(s \psi_{1} \psi_{2}\right) \otimes\left(t \psi_{1} \psi_{2}\right) \leq(s \cdot t) \psi_{1} \psi_{2}$.

Thus inductive categories and inductive category prefunctors form a category.

We now prove that the functions of Definitions 5.2 and 5.4 are indeed connected in the desired way:

Proposition 5.7. Let $\theta: S \rightarrow T$ be an ordered $(\wedge, r)$-premorphism of restriction semigroups $S$ and $T$. We define $\Theta:=\mathbf{C}(\theta): \mathbf{C}(S) \rightarrow \mathbf{C}(T)$ to be the same function on the underlying sets. Then $\Theta$ is an inductive category prefunctor with respect to the restricted products in $\mathbf{C}(S)$ and $\mathbf{C}(T)$.

Proof. (ICP1) Suppose that $\exists s \cdot t$. Then

$$
\begin{aligned}
(s \Theta) \otimes(t \Theta) & =(s \theta)(t \theta) \text { (the product in } T) \\
& \leq(s t) \theta=(s \cdot t) \theta, \text { since } \exists s \cdot t \\
& =(s \cdot t) \Theta .
\end{aligned}
$$

(ICP2) We have $\mathbf{d}(s \Theta)=(s \theta)^{+} \leq s^{+} \theta=\mathbf{d}(s) \Theta$. Similarly, $\mathbf{r}(s \Theta) \leq \mathbf{r}(s) \Theta$.

(ICP3) This follows from Lemma 4.6.

(ICP4) Let $a \in \mathbf{C}(S)$ and $f \in \mathbf{C}(S)_{o}=E$. Then

$a \Theta \mid \mathbf{r}(a \Theta) \wedge f \Theta=(a \theta)(a \theta)^{*}(f \theta)=(a \theta)(f \theta) \leq(a f) \theta=\left(a a^{*} f\right) \theta=(a \mid \mathbf{r}(a) \wedge f) \Theta$.

Similarly, $e \Theta \wedge \mathbf{d}(a \Theta) \mid a \Theta \leq(e \wedge \mathbf{d}(a) \mid a) \Theta$, for $e \in E$.

Proposition 5.8. Let $\psi: C \rightarrow D$ be an inductive category prefunctor. We define $\Psi:=\mathbf{S}(\psi): \mathbf{S}(C) \rightarrow \mathbf{S}(D)$ to be the same function on the underlying sets. Then $\Psi$ is an ordered $(\wedge, r)$-premorphism with respect to the pseudoproducts in $\mathbf{S}(C)$ and $\mathbf{S}(D)$.

Proof. $(\wedge 1)$ Let $s, t \in \mathbf{S}(C)$. Then $(s \Psi) \otimes(t \Psi)=(s \psi) \otimes(t \psi) \leq(s \otimes t) \psi=$ $(s \otimes t) \Psi$, by an argument identical to that found between lines (5.1) and (5.2) in Lemma 5.6.

$(\wedge 2)$ We have: $(s \Psi)^{+}=\mathbf{d}(s \psi) \leq \mathbf{d}(s) \psi=s^{+} \Psi$. Similarly, $(s \Psi)^{*} \leq s^{*} \Psi$.

Finally, it follows from Lemma 4.6 that $\Psi$ is order-preserving.

Once again, it is easy to see that if $\theta: S \rightarrow T$ is an ordered $(\wedge, r)$-premorphism of restriction semigroups and $\psi: C \rightarrow D$ is an inductive category prefunctor, then $\mathbf{S}(\mathbf{C}(\theta))=\theta$ and $\mathbf{C}(\mathbf{S}(\psi))=\psi$. Furthermore, if $\theta^{\prime}: T \rightarrow T^{\prime}$ is another ordered $(\wedge, r)$-premorphism of restriction semigroups, and $\psi^{\prime}: D \rightarrow D^{\prime}$ is another inductive category prefunctor of inductive categories, then $\mathbf{C}\left(\theta \theta^{\prime}\right)=\mathbf{C}(\theta) \mathbf{C}\left(\theta^{\prime}\right)$ and $\mathbf{S}\left(\phi \phi^{\prime}\right)=\mathbf{S}(\phi) \mathbf{S}\left(\phi^{\prime}\right)$. We have therefore proved Theorem 5.1 .

5.2. Strong $(\wedge, \boldsymbol{r})$-premorphisms. As we noted at the beginning of the section, in order to make the connection with the inverse case, we must now introduce the intermediate step of strong $(\wedge, r)$-premorphisms between restriction semigroups. Our goal for the remainder of this section is the proof of the following corollary to Theorem 5.1: 
Theorem 5.9. The category of restriction semigroups and strong $(\wedge, r)$-premorphisms is isomorphic to the category of inductive categories and strong inductive category prefunctors.

Once again, it only remains to deal with the 'arrows' part.

In the study of partial actions of one-sided restriction semigroups [10], it is the notion of a 'strong premorphism' which has proved most useful. We make the following definition in the two-sided case:

Definition 5.10. Let $S$ and $T$ be restriction semigroups. A $(\wedge, r)$-premorphism $\theta: S \rightarrow T$ is called strong if

$$
(\wedge 1)^{\prime}(s \theta)(t \theta)=(s \theta)^{+}(s t) \theta=(s t) \theta(t \theta)^{*} .
$$

It is clear that condition $(\wedge 1)$ follows from condition $(\wedge 1)^{\prime}$, by $(2.2)$. Furthermore, we deduce the following from [10, Lemma 2.10(4)]:

Lemma 5.11. A strong $(\wedge, r)$-premorphism $\theta: S \rightarrow T$ between restriction semigroups is order-preserving.

We therefore drop all explicit mention of order-preservation from here on.

Lemma 5.12. The composition of two strong $(\wedge, r)$-premorphisms is a strong $(\wedge, r)$-premorphism.

Proof. Let $\theta_{1}: U \rightarrow V$ and $\theta_{2}: V \rightarrow W$ be strong $(\wedge, r)$-premorphisms of restriction semigroups $U, V$ and $W$. Condition $(\wedge 2)$ is immediate: $\left(s \theta_{1} \theta_{2}\right)^{+} \leq$ $\left(\left(s \theta_{1}\right)^{+}\right) \theta_{2} \leq s^{+} \theta_{1} \theta_{2}$. Similarly, $\left(s \theta_{1} \theta_{2}\right)^{*} \leq s^{*} \theta_{1} \theta_{2}$.

For $(\wedge 1)^{\prime}$, we have $\left(s \theta_{1} \theta_{2}\right)\left(t \theta_{1} \theta_{2}\right)=\left(s \theta_{1} \theta_{2}\right)^{+}\left(\left(s \theta_{1}\right)\left(t \theta_{1}\right)\right) \theta_{2}$, using $(\wedge 1)^{\prime}$ for $\theta_{2}$. Now,

$$
\begin{aligned}
\left(s \theta_{1} \theta_{2}\right)^{+} \leq\left(\left(s \theta_{1}\right)^{+}\right) \theta_{2} & \Longrightarrow\left(s \theta_{1} \theta_{2}\right)^{+} \leq\left(\left(s \theta_{1}\right)^{+}\right) \theta_{2}^{+} \\
& \Longrightarrow\left(s \theta_{1} \theta_{2}\right)^{+}=\left(s \theta_{1} \theta_{2}\right)^{+}\left(\left(s \theta_{1}\right)^{+}\right) \theta_{2}^{+},
\end{aligned}
$$

by $(2.3)$, so

$$
\begin{aligned}
\left(s \theta_{1} \theta_{2}\right)\left(t \theta_{1} \theta_{2}\right) & =\left(s \theta_{1} \theta_{2}\right)^{+}\left(\left(s \theta_{1}\right)^{+}\right) \theta_{2}^{+}\left(\left(s \theta_{1}\right)\left(t \theta_{1}\right)\right) \theta_{2} \\
& =\left(s \theta_{1} \theta_{2}\right)^{+}\left(\left(s \theta_{1}\right)^{+}\right) \theta_{2}^{+}\left(\left(s \theta_{1}\right)^{+}(s t) \theta_{1}\right) \theta_{2}
\end{aligned}
$$

(using $(\wedge 1)^{\prime}$ for $\left.\theta_{1}\right)$

$$
=\left(s \theta_{1} \theta_{2}\right)^{+}\left(\left(s \theta_{1}\right)^{+}\right) \theta_{2}(s t) \theta_{1} \theta_{2}
$$

(applying $\left(a \theta_{2}\right)\left(b \theta_{2}\right)=\left(a \theta_{2}\right)^{+}(a b) \theta_{2}$ with $a=\left(s \theta_{1}\right)^{+}$and $\left.b=(s t) \theta_{1}\right)$

$$
=\left(s \theta_{1} \theta_{2}\right)^{+}(s t) \theta_{1} \theta_{2},
$$

since $\left(s \theta_{1} \theta_{2}\right)^{+} \leq\left(\left(s \theta_{1}\right)^{+}\right) \theta_{2}$. Similarly, $\left(s \theta_{1} \theta_{2}\right)\left(t \theta_{1} \theta_{2}\right)=(s t) \theta_{1} \theta_{2}\left(s \theta_{1} \theta_{2}\right)^{*}$. 
Thus restriction semigroups together with strong $(\wedge, r)$-premorphisms form a category.

We now define a function between inductive categories which will correspond to a strong $(\wedge, r)$-premorphism between restriction semigroups.

Definition 5.13. An inductive category prefunctor $\psi: C \rightarrow D$ will be called strong if

(ICP5) (a) $\mathbf{d}((s \psi) \otimes(t \psi))=\mathbf{d}(s \psi) \wedge \mathbf{d}((s \otimes t) \psi)$;

(b) $\mathbf{r}((s \psi) \otimes(t \psi))=\mathbf{r}((s \otimes t) \psi) \wedge \mathbf{r}(t \psi)$.

We note the following:

Lemma 5.14. Condition (ICP4) follows from (ICP5).

Proof. Let $\psi: C \rightarrow D$ be a function between inductive categories which satisfies conditions (ICP1)-(ICP3), plus (ICP5). We consider (ICP4)'(a) and observe that, for $a \in C$ and $f \in C_{o}$,

$$
\begin{aligned}
a \psi \otimes f \psi \leq(a \otimes f) \psi \text { in } D & \Longleftrightarrow a \psi \otimes f \psi \leq(a \otimes f) \psi \text { in } \mathbf{S}(D) \\
& \Longleftrightarrow a \psi \otimes f \psi=(a \otimes f) \psi \otimes \mathbf{r}(a \psi \otimes f \psi) \\
& \Longleftrightarrow a \psi \otimes f \psi=(a \otimes f) \psi \otimes(\mathbf{r}(a \psi) \wedge f \psi),
\end{aligned}
$$

using Lemma 3.7. We will use Lemma 3.10, in conjunction with (ICP5), to demonstrate the equality (5.3). We note first of all that $a \psi \otimes f \psi \leq a \psi$ and also that

$$
(a \otimes f) \psi \otimes(\mathbf{r}(a \psi) \wedge f \psi) \leq(a \otimes f) \psi \leq a \psi,
$$

since $a \otimes f \leq a$ and $\psi$ is order-preserving. It remains to show that each side of (5.3) has the same range. On the one hand, we have

$$
\mathbf{r}(a \psi \otimes f \psi)=\mathbf{r}((a \otimes f) \psi) \wedge \mathbf{r}(f \psi)=\mathbf{r}((a \otimes f) \psi) \wedge f \psi,
$$

by (ICP5)(b). On the other, using Lemma 3.7,

$$
\begin{aligned}
(a \otimes f) \psi \otimes(\mathbf{r}(a \psi) \wedge f \psi) & =(a \otimes f) \psi \mid \mathbf{r}((a \otimes f) \psi) \wedge \mathbf{r}(a \psi) \wedge f \psi, \\
& =(a \otimes f) \psi \mid \mathbf{r}((a \otimes f) \psi) \wedge f \psi,
\end{aligned}
$$

since $\mathbf{r}((a \otimes f) \psi) \leq \mathbf{r}(a \psi)$. Thus

$$
\mathbf{r}((a \otimes f) \psi \otimes(\mathbf{r}(a \psi) \wedge f \psi))=\mathbf{r}((a \otimes f) \psi) \wedge f \psi=\mathbf{r}(a \psi \otimes f \psi),
$$

as required. We conclude that (ICP4)'(a) holds. Part (b) follows similarly.

Thus a strong inductive category prefunctor may be regarded as a function defined by conditions (ICP1)-(ICP3) and (ICP5) only.

We defer the proof that the composition of two strong inductive category prefunctors is a strong inductive category prefunctor until after the following propositions, which prove that the functions of Definitions 5.10 and 5.13 are once again connected in the desired way: 
Proposition 5.15. Let $\theta: S \rightarrow T$ be a strong $(\wedge, r)$-premorphism of restriction semigroups. We define $\Theta:=\mathbf{C}(\theta): \mathbf{C}(S) \rightarrow \mathbf{C}(T)$ to be the same function on the underlying sets. Then $\Theta$ is a strong inductive category prefunctor with respect to the restricted products in $\mathbf{C}(S)$ and $\mathbf{C}(T)$.

Proof. By Proposition 5.7, $\Theta$ is an inductive category prefunctor.

(ICP5)(a) We have

$$
\begin{aligned}
\mathbf{d}((s \Theta) \otimes(t \Theta)) & =((s \theta)(t \theta))^{+}=\left((s \theta)^{+}(s t) \theta\right)^{+}=\left((s \theta)^{+}(s t) \theta^{+}\right)^{+} \\
& =(s \theta)^{+}(s t) \theta^{+}=\mathbf{d}(s \Theta) \wedge \mathbf{d}((s \otimes t) \Theta) .
\end{aligned}
$$

Part (b) is similar.

Proposition 5.16. Let $\psi: C \rightarrow D$ be a strong inductive category prefunctor. We define $\Psi:=\mathbf{S}(\psi): \mathbf{S}(C) \rightarrow \mathbf{S}(D)$ to be the same function on the underlying sets. Then $\Psi$ is a strong $(\wedge, r)$-premorphism with respect to the pseudoproducts in $\mathbf{S}(C)$ and $\mathbf{S}(D)$.

Proof. By Proposition 5.8, $\Psi$ is an ordered $(\wedge, r)$-premorphism.

We know that $(s \Psi) \otimes(t \Psi) \leq(s \otimes t) \Psi$ and that $(s \Psi)^{+} \otimes(s \otimes t) \Psi \leq(s \otimes t) \Psi$, by (2.2). We will use Lemma 3.10 to show that condition $(\wedge 1)^{\prime}$ holds. It remains to show that both sides of the desired equality have the same domain. From (ICP5), we have:

$$
\begin{aligned}
\mathbf{d}((s \Psi) \otimes(t \Psi)) & =\mathbf{d}(s \Psi) \wedge \mathbf{d}((s \otimes t) \Psi)=\mathbf{d}(\mathbf{d}(s \Psi) \wedge \mathbf{d}((s \otimes t) \Psi)) \\
& =\left((s \Psi)^{+} \otimes(s \otimes t) \Psi^{+}\right)^{+}=\left((s \Psi)^{+} \otimes(s \otimes t) \Psi\right)^{+} \\
& =\mathbf{d}\left((s \Psi)^{+} \otimes(s \otimes t) \Psi\right) .
\end{aligned}
$$

So by Lemma 3.10, $(s \Psi) \otimes(t \Psi)=(s \Psi)^{+} \otimes(s \otimes t) \Psi$. By a similar argument, $(s \Psi) \otimes(t \Psi)=(s \otimes t) \Psi \otimes(t \Psi)^{*}$.

It is clear that if $\theta: S \rightarrow T$ is a strong $(\wedge, r)$-premorphism and $\psi: C \rightarrow T$ is a strong inductive category prefunctor, then $\mathbf{S}(\mathbf{C}(\theta))=\theta$ and $\mathbf{C}(\mathbf{S}(\psi))=$ $\psi$. Furthermore, if $\theta^{\prime}: T \rightarrow T^{\prime}$ is another strong $(\wedge, r)$-premorphism, then $\mathbf{C}\left(\theta \theta^{\prime}\right)=\mathbf{C}(\theta) \mathbf{C}\left(\theta^{\prime}\right)$. In order to complete the proof of Theorem 5.9, we must prove that the composition of two strong inductive category prefunctors is also a strong inductive category prefunctor, thereby showing that inductive categories together with strong inductive category prefunctors do indeed form a category.

Lemma 5.17. The composition of two strong inductive category prefunctors is a strong inductive category prefunctor.

Proof. We take an indirect approach using the preceding proposition. Let $\psi_{1}$ : $U \rightarrow V$ and $\psi_{2}: V \rightarrow W$ be strong inductive category prefunctors between inductive categories $U, V$ and $W$. By Proposition [5.16, we can construct strong $(\wedge, r)$-premorphisms $\mathbf{S}\left(\psi_{1}\right): \mathbf{S}(U) \rightarrow \mathbf{S}(V)$ and $\mathbf{S}\left(\psi_{2}\right): \mathbf{S}(V) \rightarrow \mathbf{S}(W)$. Then $\mathbf{S}\left(\psi_{1}\right) \mathbf{S}\left(\psi_{2}\right): \mathbf{S}(U) \rightarrow \mathbf{S}(W)$ is a strong $(\wedge, r)$-premorphism, by Lemma 5.12 . 
Now, $\psi_{1} \psi_{2}$ is certainly an inductive category prefunctor (but is not necessarily strong), in which case, $\mathbf{S}\left(\psi_{1} \psi_{2}\right)$ is an ordered $(\wedge, r)$-premorphism, by Proposition 5.8. Let ' $\sim$ ' denote the relationship '...is the same function on the underlying sets as...'. Then $\psi_{1} \sim \mathbf{S}\left(\psi_{1}\right)$ and $\psi_{2} \sim \mathbf{S}\left(\psi_{2}\right)$, so $\psi_{1} \psi_{2} \sim \mathbf{S}\left(\psi_{1}\right) \mathbf{S}\left(\psi_{2}\right)$. But $\psi_{1} \psi_{2} \sim \mathbf{S}\left(\psi_{1} \psi_{2}\right)$. We deduce that $\mathbf{S}\left(\psi_{1}\right) \mathbf{S}\left(\psi_{2}\right)=\mathbf{S}\left(\psi_{1} \psi_{2}\right)$, hence $\mathbf{S}\left(\psi_{1} \psi_{2}\right)$ is a strong $(\wedge, r)$-premorphism. Then $\mathbf{C}\left(\mathbf{S}\left(\psi_{1} \psi_{2}\right)\right)=\psi_{1} \psi_{2}$ is a strong inductive category prefunctor, by Proposition 5.15 .

Thus Theorem 5.9 is proved.

\section{The INVERSE CASE}

We will now deduce a corollary to Theorem 5.9 in the inverse case. Our notion of ' $\wedge$-premorphism' will simply be that of Definition 1.2. In addition, if such a $(\wedge, i)$-premorphism is order-preserving, then we will call it an ordered $(\wedge, i)$ premorphism. We will prove the following:

Theorem 6.1. The category of inverse semigroups and ordered $(\wedge, i)$-premorphisms is isomorphic to the category of inductive groupoids and ordered groupoid premorphisms.

Note that the 'objects' part of the above theorem is taken care of by the comments at the end of Section 4 .

We deduce the following from [10, Lemma 2.12]:

Lemma 6.2. Let $\theta: S \rightarrow T$ be a mapping between inverse semigroups. Then $\theta$ is an ordered $(\wedge, i)$-premorphism if, and only if, it is a strong $(\wedge, r)$-premorphism of restriction semigroups.

We now, of course, need a corresponding function between inductive groupoids. In his study of the partial actions of inductive groupoids, Gilbert [7] has employed the following definition:

Definition 6.3. [7, p. 184] A function $\psi: G \rightarrow H$ between inductive groupoids will be called an ordered groupoid premorphism if the following conditions are satisfied:

(ICP1) if the product $g \cdot h$ is defined in $G$, then $(g \psi) \otimes(h \psi) \leq(g \cdot h) \psi$;

(IGP) $(g \psi)^{-1}=g^{-1} \psi$;

(ICP3) if $g \leq h$ in $G$, then $g \psi \leq h \psi$ in $H$.

We want to show that Gilbert's ordered groupoid premorphisms are a special case of our strong inductive category prefunctors. In particular, as a first step towards establishing an analogue of Lemma 6.2, we need to show that an ordered groupoid premorphism satisfies condition (ICP5). Unfortunately, an elementary proof of this does not seem to be forthcoming; instead, we provide a proof which employs the machinery of the Szendrei expansion of an inductive groupoid, as introduced in Section 3.2, together with the following result of Gilbert: 
Theorem 6.4. [7, Theorem 4.4 \& Proposition 4.6] Let $G$ and $H$ be inductive groupoids. If $\psi: G \rightarrow H$ is an ordered groupoid premorphism, then there exists a unique inductive functor $\bar{\psi}: \mathrm{Sz}(G) \rightarrow H$ such that $\iota \bar{\psi}=\psi$. Conversely, if $\bar{\psi}: \mathrm{Sz}(G) \rightarrow H$ is an inductive functor, then $\psi:=\iota \bar{\psi}$ is an ordered groupoid premorphism.

Lemma 6.5. Let $\psi: G \rightarrow H$ be an ordered groupoid premorphism. Then $\psi$ satisfies condition (ICP5).

Proof. By Theorem 6.4, $\psi$ may be decomposed as $\psi=\imath \bar{\psi}$, where $\iota: G \rightarrow \operatorname{Sz}(G)$ is as in Section 3.2 and $\bar{\psi}: \mathrm{Sz}(G) \rightarrow H$ is an inductive functor. Note that an inductive functor preserves products, domains, ranges, ordering, restrictions, corestrictions and meets. We will demonstrate that (ICP5)(a) holds; part (b) is similar. Let $s, t \in G$. Then $s \psi=s \iota \bar{\psi}=(\{\mathbf{d}(s), s\}, s) \bar{\psi}$ and $t \psi=t \iota \bar{\psi}=$ $(\{\mathbf{d}(t), t\}, t) \bar{\psi}$. We have

$$
\begin{aligned}
s \psi \otimes t \psi & =(\{\mathbf{d}(s), s\}, s) \bar{\psi} \otimes(\{\mathbf{d}(t), t\}, t) \bar{\psi} \\
& =[(\{\mathbf{d}(s), s\}, s) \otimes(\{\mathbf{d}(t), t\}, t)] \bar{\psi} \\
& =(\Delta \mid\{\mathbf{d}(s), s\} \cup s \otimes\{\mathbf{d}(t), t\}, s \otimes t) \bar{\psi},
\end{aligned}
$$

by (3.3), where $\Delta=\mathbf{d}(s \mid \mathbf{r}(s) \wedge \mathbf{d}(t))=\mathbf{d}(s \otimes t)$. Now, $\Delta \leq \mathbf{d}(s)$, so $\Delta \mid \mathbf{d}(s)=\Delta$. Also, $s \otimes \mathbf{d}(t)=s|\mathbf{r}(s) \wedge \mathbf{d}(t)=\Delta| s$, by Lemma 3.10, so

$$
s \psi \otimes t \psi=(\{\Delta, \Delta \mid s, s \otimes t\}, s \otimes t) \bar{\psi}
$$

Then, by (3.1),

$$
\mathbf{d}(s \psi \otimes t \psi)=(\{\Delta, \Delta \mid s, s \otimes t\}, \Delta) \bar{\psi}
$$

On the other hand,

$$
(s \otimes t) \psi=(s \otimes t) \iota \bar{\psi}=(\{\mathbf{d}(s \otimes t), s \otimes t\}, s \otimes t) \bar{\psi}=(\{\Delta, s \otimes t\}, s \otimes t) \bar{\psi},
$$

so that

$$
\mathbf{d}((s \otimes t) \psi)=(\{\Delta, s \otimes t\}, \Delta) \bar{\psi}
$$

Note that $\mathbf{d}(s \psi)=(\{\mathbf{d}(s), s\}, \mathbf{d}(s)) \bar{\psi}$. We have

$$
\begin{aligned}
\mathbf{d}(s \psi) \wedge \mathbf{d}((s \otimes t) \psi) & =(\{\mathbf{d}(s), s\}, \mathbf{d}(s)) \bar{\psi} \wedge(\{\Delta, s \otimes t\}, \Delta) \bar{\psi} \\
& =[(\{\mathbf{d}(s), s\}, s), \mathbf{d}(s)) \wedge(\{\Delta, s \otimes t\}, \Delta)] \bar{\psi} \\
& =(\Delta \mid\{\mathbf{d}(s), s, \Delta, s \otimes t\}, \Delta) \bar{\psi} \quad(\text { by (3.2) }) \\
& =(\{\Delta, \Delta \mid s, s \otimes t\}, \Delta) \bar{\psi}
\end{aligned}
$$

(since $\Delta \mid(s \otimes t)=s \otimes t$, by Lemma 3.4)

$$
=\mathbf{d}(s \psi \otimes t \psi)
$$

as required.

We note the following: 
Lemma 6.6. [7, Lemma 4.2] Let $\psi: G \rightarrow H$ be an ordered groupoid premorphism. Then, for any $g \in G, \mathbf{d}(g \psi) \leq \mathbf{d}(g) \psi$ and $\mathbf{r}(g \psi) \leq \mathbf{r}(g) \psi$.

That is, an ordered groupoid premorphism satisfies condition (ICP2). Thus, by the two preceding lemmas, every ordered groupoid premorphism is a strong inductive category prefunctor.

Lemma 6.7. The composition of two ordered groupoid premorphisms is an ordered groupoid premorphism.

Proof. The composition of two ordered groupoid premorphisms is certainly a strong inductive category prefunctor, by Lemma 5.17. Condition (IGP) follows easily.

Inductive groupoids together with ordered groupoid premorphisms therefore form a category.

Proposition 6.8. Let $\theta: S \rightarrow T$ be an ordered $(\wedge, i)$-premorphism. We define $\Theta:=\mathbf{C}(\theta): \mathbf{C}(S) \rightarrow \mathbf{C}(T)$ to be the same function on the underlying sets. Then $\Theta$ is an ordered groupoid premorphism with respect to the restricted products in $\mathbf{C}(S)$ and $\mathbf{C}(T)$.

Proof. By Lemma 6.2, $\theta$ is a strong $(\wedge, r)$-premorphism. Then $\Theta$ is a strong inductive category prefunctor, by Proposition 5.15. It remains to show that $\Theta$ satisfies (IGP): $(g \Theta)^{-1}=(g \theta)^{-1}=g^{-1} \theta=g^{-1} \Theta$.

Proposition 6.9. Let $\psi: G \rightarrow H$ be an ordered groupoid premorphism. We define $\Psi:=\mathbf{S}(\psi): \mathbf{S}(G) \rightarrow \mathbf{S}(H)$ to be the same function on the underlying sets. Then $\Psi$ is an ordered $(\wedge, i)$-premorphism with respect to the pseudoproducts in $\mathbf{S}(G)$ and $\mathbf{S}(H)$.

Proof. We know that $\psi$ is a strong inductive category prefunctor. Then, by Proposition 5.16, $\Psi$ is a strong $(\wedge, r)$-premorphism. It remains to show that condition $(\wedge 2)^{\prime}$ is satisfied: $(g \Psi)^{-1}=(g \psi)^{-1}=g^{-1} \psi=g^{-1} \Psi$, as required.

Thus ordered groupoid premorphisms and ordered $(\wedge, i)$-premorphisms are connected in the manner required to prove Theorem 6.1. We can now complete our inductive groupoid analogue of Lemma 6.2;

Lemma 6.10. Let $\psi: G \rightarrow H$ be a function between inductive groupoids. Then $\psi$ is an ordered groupoid premorphism if, and only if, it is a strong inductive category prefunctor.

Proof. $(\Rightarrow)$ Suppose that $\psi: G \rightarrow H$ is an ordered groupoid premorphism. It follows from Lemmas 6.5 and 6.6 that $\psi$ is a strong inductive category prefunctor.

$(\Leftarrow)$ Suppose that $\psi: G \rightarrow H$ is a strong inductive category prefunctor of inductive groupoids. By Proposition 5.16, we have a strong $(\wedge, r)$-premorphism $\mathbf{S}(\psi): \mathbf{S}(G) \rightarrow \mathbf{S}(H)$. But this is a strong $(\wedge, r)$-premorphism of inverse semigroups, so, by Lemma 6.2, it is an ordered $(\wedge, i)$-premorphism. Then $\mathbf{C}(\mathbf{S}(\psi))=$ $\psi$ is an ordered groupoid premorphism, by Proposition 6.8. 
As ever, it is clear that if $\theta: S \rightarrow T$ is an ordered $(\wedge, i)$-premorphism and $\psi: G \rightarrow H$ is an ordered groupoid premorphism, then $\mathbf{S}(\mathbf{C}(\theta))=\theta$ and $\mathbf{C}(\mathbf{S}(\psi))=\psi$. Also, if $\theta^{\prime}: T \rightarrow T^{\prime}$ is another ordered $(\wedge, i)$-premorphism of inverse semigroups, and $\psi^{\prime}: H \rightarrow H^{\prime}$ is another ordered groupoid premorphism, then $\mathbf{C}\left(\theta \theta^{\prime}\right)=\mathbf{C}(\theta) \mathbf{C}\left(\theta^{\prime}\right)$ and $\mathbf{S}\left(\psi \psi^{\prime}\right)=\mathbf{S}(\psi) \mathbf{S}\left(\psi^{\prime}\right)$. We have proved Theorem 6.1.

Let REST denote a category whose objects are restriction semigroups; subscripts of 'mor', 'strong', ' $\wedge$ ' and ' $\vee$ ' will denote that the arrows of the category are $(2,1,1)$-morphisms, strong $(\wedge, r)$-premorphisms, ordered $(\wedge, r)$-premorphisms and $(\vee, r)$-premorphisms, respectively. Thus, for example, $\mathbf{R E S T}_{\text {strong }}$ denotes the category of restriction semigroups and strong $(\wedge, r)$-premorphisms. Similarly, INV will denote a category whose objects are inverse semigroups; the subscripts applied here will be 'mor', ' $\wedge$ ' and ' $\vee$ ', denoting morphisms, ordered $(\wedge, i)$-premorphisms and $(\vee, i)$-premorphisms, respectively. A category whose objects are inductive categories or inductive groupoids will be denoted by $\mathbf{I C}$ or $\mathbf{I G}$, as appropriate. A subscript of 'ord' or 'ind' will denote ordered functors or inductive functors, respectively, whilst 'pre', 'strong' and 'ogp' will denote inductive category prefunctors, strong inductive category prefunctors and ordered groupoid premorphisms, respectively. We can now summarise the connections between the various categories in this paper in the following pair of Hasse diagrams:
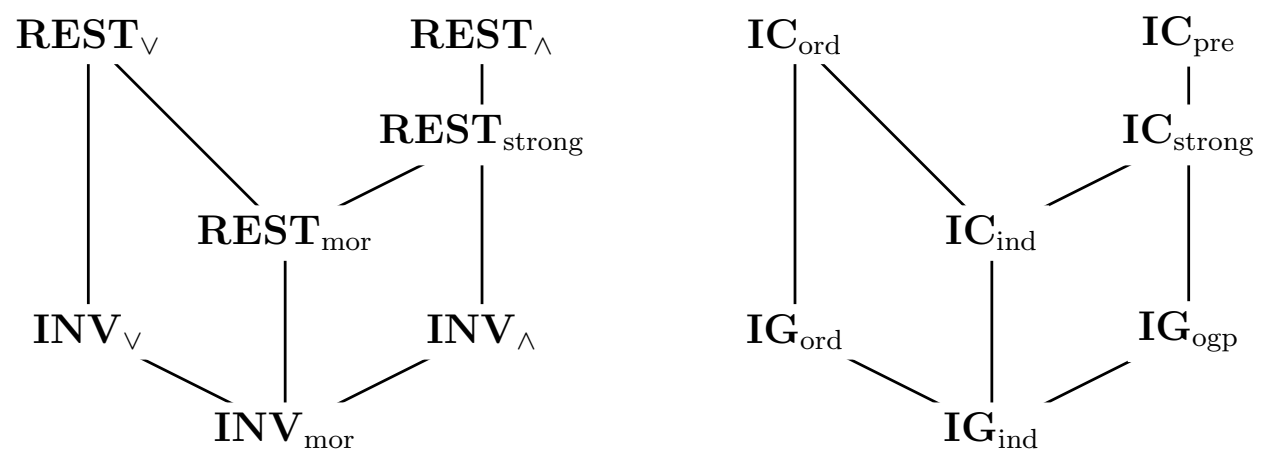

Each category in the left-hand diagram is isomorphic to the corresponding category in the right-hand diagram, and vice versa.

\section{REFERENCES}

[1] S. Armstrong, The structure of type A semigroups, Semigroup Forum, 29 (1984), 319-336.

[2] J.-C. Birget and J. Rhodes, Almost finite expansions of arbitrary semigroups, Journal of Pure and Applied Algebra, 32 (1984), 239-287.

[3] J. R. B. Cockett and E. Manes, Boolean and classical restriction categories, preprint, 2007.

[4] J. Fountain, A class of right PP monoids, Quarterly Journal of Mathematics, Oxford (2), 28 (1977), 285-300.

[5] J. Fountain, Adequate semigroups, Proceedings of the Edinburgh Mathematical Society (2), 22 (1979), 113-125. 
[6] J. Fountain and G. M. S. Gomes, The Szendrei expansion of a semigroup, Mathematika, 37 (1990), 251-260.

[7] N. D. Gilbert, Actions and expansions of ordered groupoids, Journal of Pure and Applied Algebra, 198 (2005), 175-195.

[8] G. M. S. Gomes and V. Gould, Finite proper covers in a class of finite semigroups with commuting idempotents, Semigroup Forum, 66 (2003), 433-454.

[9] V. Gould, (Weakly) left $E$-ample semigroups, http://www-users.york.ac.uk/ varg1/finitela.ps

[10] V. Gould and C. Hollings, Partial actions of inverse and weakly left $E$-ample semigroups, to appear in Journal of the Australian Mathematical Society.

[11] C. Hollings, Partial Actions of Semigroups and Monoids, Ph.D. thesis, University of York, 2007.

[12] C. Hollings, Partial actions of monoids, Semigroup Forum, 75(2) (2007), 293-316.

[13] M. Jackson and T. Stokes, An invitation to $C$-semigroups, Semigroup Forum, 62 (2001), 279-310.

[14] N. Jacobson, Basic Algebra, Volume II, W. H. Freeman and Co., San Francisco, 1980.

[15] M. V. Lawson, Semigroups and ordered categories I: the reduced case, Journal of Algebra, 141 (1991), 422-462.

[16] M. V. Lawson, Inverse Semigroups: The Theory of Partial Symmetries, World Scientific, 1998.

[17] D. B. McAlister and N. R. Reilly, E-unitary covers for inverse semigroups, Pacific Journal of Mathematics, 68 (1977), 161-174.

[18] M. B. Szendrei, A note on Birget-Rhodes expansion of groups, Journal of Pure and Applied Algebra, 58 (1989), 93-99.

Centro de Álgebra da Universidade de Lisboa, Av. Prof. Gama Pinto 2, 1649003 Lisboa, Portugal

E-mail address: cdh500@cii.fc.ul.pt 